\title{
Continuum modeling of beamlike lattice trusses using averaging methods
}

\author{
B. Burgardt, P. Cartraud \\ Department of Mechanics and Materials, Ecole Centrale de Nantes, B.P. 92101, 44321, Nantes, Cedex 3, France
}

A general procedure to determine the equivalent beam properties of beam-like lattice trusses is presented. The method is based on the energy equivalence. Its main features are the use of piecewise linear functions to represent the displacements, and the definition of the continuum stress and strain parameters by their average values over the continuum cell. This allows a unifying approach to be obtained to derive methods for computing the effective beam properties. It is shown that there is only one rigorous method, and this method takes the lattice periodicity into account. Moreover, the classical method based on static condensation is found to be only approximate. The procedure is applied to examples of planar lattice trusses in static analysis. The results prove the effectiveness and the reliability of the present approach, and comparisons are made with results obtained from other classical methods.

Keywords: Truss structures; Continuum modeling; Beam-like structures; Homogenization

\section{Introduction}

Lattice structures have been used for many years in civil engineering, and they are candidates for future applications in space, due to their low cost, light weight and high stiffness. A discrete finite element model of a large repetitive structure can become computationally expensive, and inappropriate to study the overall structural response and the vibration modes. This is why simplified models have to be developed.

Several methods have been discussed in the literature, and they are reviewed in the survey paper [1]. Among them, the substitute continuum approach has several advantages, and has proved to be suitable for the analysis of large repetitive lattices [1]. In this field, the approaches used can be grouped into two main classes.

The first one includes methods in which the continuum behaviour is assumed a priori (e.g. a Timoshenko-beam for a beam-like lattice). Then, the problem amounts to finding the equivalent continuum properties. The equivalence can be defined on the energies [1-5], or on the constitutive relations, by transforming the force-displacement relationships in the repetitive lattice into constitutive equations in the continuum model [6].

In the second class of methods, the governing laws of the continuum are obtained from the discrete nodal equations. Then, either an asymptotic expansion and a double-scale writing [7], or a finite difference operator relating the displacements of two consecutive lattice sections [8] are used, or both [9].

In this paper, the general framework of the energy equivalence between the lattice and the continuum 
model is used to derive the equivalent properties. Usually, the main steps of such an approach consist of [1-4]:

1. Isolating a typical (repeating) cell.

2. Expanding the nodal displacements in a Taylor series.

3. Identifying the strain parameters for the continuum.

4. Transforming the energies expressed in the nodal quantities into continuum displacements and strains.

5. Computing the effective continuum properties.

The method presented herein follows this procedure, but with changes in steps 2, 3 and 5. A new displacement field expression is introduced based on piecewise linear functions in order to describe the exact displacement field in the trusses more accurately. Besides, in connection with the homogenization theory of periodic materials [10], the continuum strain and stress parameters are defined by their average values over the cell. The characterization of the continuum stresses represents the most significant improvement to the methods based on the energy equivalence proposed in the literature [1-5]. Then, a rigorous partitioning of the strain parameters is possible, so as to derive the equivalent continuum properties, and take the lattice periodicity into account. Moreover, from the matrix formulation obtained, it will be shown that the static condensation which is widely used [2-5] to obtain the effective continuum properties, is an approximation.

In the present paper, the method is applied to plane pin-jointed beam-like lattices in static analysis, but it can also be used for three-dimensional lattices in dynamic analysis.

The expressions for the displacement field and the definition of the continuum strain and stress parameters are presented in Section 2. From the matrix formulation in Section 3, different methods to determine the constitutive relations for the Timoshenkobeam model are proposed in Section 4, and comparisons between the methods are made in Section 5 . Finally, numerical examples are given in Section 6 and prove the reliability of our approach.

\section{Preliminary developments}

\subsection{Expressions of displacements}

The starting point for the continuum modeling of lattice trusses is usually the introduction of a displacement field, which is herein based on the representation of the deformed shape of the bay boundary. In order to illustrate our approach, let us first consider a singlebay lattice (only one bay on the cell length). The case of the double-bay lattice will be treated afterwards.

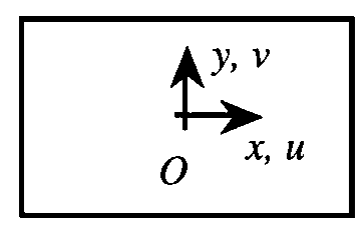

Fig. 1. The single-bay lattice.

\subsubsection{Single-bay lattice}

A study is made of the planar rectangle of length $l$ and height $h$ shown in Fig. 1, with the origin of the coordinate system at the cell center.

All the truss members are pin-jointed, and the cell can either be a single- or a double-laced lattice truss (only the vertical and the horizontal trusses have been represented). Then, a finite element model introduces 8 degrees of freedom.

It can be seen that the following displacement field expressions $((x, y)$ are the coordinates of a truss point):

$u(x, y)=a_{0}+a_{1} x+a_{2} y+a_{3} x y$

$v(x, y)=b_{0}+b_{1} x+b_{2} y+b_{3} x y$

gives an exact representation of the deformed shape of the bay boundary. Therefore, the number of parameters introduced is equal to the total number of degrees of freedom of the cell.

Moreover, in each truss, the displacement field is linear, and if we consider the two vertical (respectively horizontal) trusses, the axial displacement $v$ (respectively $u$ ) varies linearly in $y$ (respectively in $x$ ). As a result, Eq. (1) gives an exact description of the displacement field for these trusses parallel to the coordinate axes. However, for the diagonal trusses, the axial displacement is found from Eq. (1) to be an approximation in the form of a polynomial of second degree in $x^{\prime}$, the local abscissa of the truss.

If the continuum which completely covers the cell is considered, the displacement field in it is also given by Eq. (1), and is extended to the continuum domain, with $(x, y) \in S_{\mathrm{c}}=[-l / 2, l / 2] \times[-h / 2, h / 2]$.

After differentiation, one can easily obtain (the subscript,$x$ indicates a partial derivative with respect to $x)$ :

$u(x, y)=u_{0}+x \epsilon_{x}+y\left(\frac{1}{2} \gamma_{x y}-r_{0}\right)+x y \epsilon_{x, y}$

$v(x, y)=v_{0}+x\left(\frac{1}{2} \gamma_{x y}+r_{0}\right)+y \epsilon_{y}+x y \epsilon_{y, x}$

where the rigid body displacement $\left(u_{0}, v_{0}\right)$, the rotation $r_{0}=\left(v_{, x}-u_{, y}\right) / 2$ and the strains are evaluated at the cell origin. 


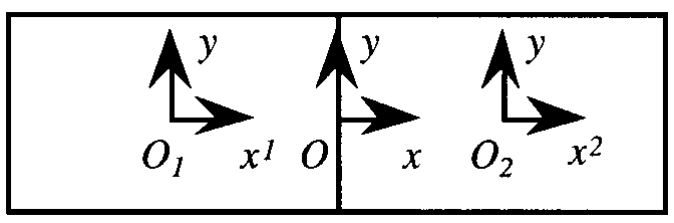

Fig. 2. The double-bay lattice.

\subsubsection{Double-bay lattice}

Let us now add a second bay (of the same length for the sake of simplicity) to the previous single-bay lattice, as shown in Fig. 2, where $O_{i}$ is the origin and $\left(x^{i}, y\right)$ the coordinates of an $i$ th bay point.

The length of the cell is again noted, $l$, with each bay of length $l / 2$. The displacement field of this double-bay lattice is simply written from the results of the previous section, considering each bay separately, hence Eq. (2), with eight parameters in each bay. The number of parameters is easily reduced to 12 (the number of degrees of freedom of the cell) and the continuity of the displacement field at the junction of the two bays is taken into account. The following equations are then obtained:

$u_{0}^{1}+\frac{l}{4} \epsilon_{x}^{1}=u_{0}^{2}-\frac{l}{4} \epsilon_{x}^{2}$

$v_{0}^{1}+\frac{l}{4}\left(\frac{1}{2} \gamma_{x y}^{1}+r_{0}^{1}\right)=v_{0}^{2}-\frac{l}{4}\left(\frac{1}{2} \gamma_{x y}^{2}+r_{0}^{2}\right)$

$\frac{1}{2} \gamma_{x y}^{1}-r_{0}^{1}+\frac{l}{4} \epsilon_{x, y}^{1}=\frac{1}{2} \gamma_{x y}^{2}-r_{0}^{2}-\frac{l}{4} \epsilon_{x, y}^{2}$

$\epsilon_{y}^{1}+\frac{l}{4} \epsilon_{y, x}^{1}=\epsilon_{y}^{2}-\frac{l}{4} \epsilon_{y, x}^{2}$

where the superscript $i$ stands for the bay $i$.

The previous displacement field expression has the main advantage of exactly representing the deformed shape of the bay boundary and the displacement in the trusses parallel to the coordinate axes, as is also the case for the single-bay lattice. This displacement is approximate only for the other trusses.

\subsubsection{Comparison with other methods}

In the literature, the problem of finding the displacement field is generally treated by means of Taylor series expansion in the axial direction of the equivalent beam. This expansion can be made directly on the displacement field [3-4], or on the strain field [1-2]. In both cases, the method consists of representing the displacements by polynomials, the order of which is dependent on the cell configuration.

For a single-bay lattice, such an approach leads to a linear displacement field, equivalent to that given in
Eq. (1) (see Ref. [3], where the rigid body rotation is expressed with a parameter different from $r_{0}$ ).

In the case of a double-bay lattice, the Taylor series expansion method leads to quadratic terms in $x$ in the displacement field expression [4]. It turns out that the deformed shape of the bay boundary is approximated, while it is exactly described by the piecewise linear functions proposed in this paper.

Therefore, it can be concluded that, for a multiplebay lattice, our approach gives a more accurate approximation of the displacement field.

\subsection{Macroscopic strains}

The next step to building a substitutive continuum model is to define the strains associated with the overall behaviour of the cell. As in the homogenization methods of periodic materials [10], we choose to work with the average values of the local strains over the continuum cell.

\subsubsection{Single-bay lattice}

Since the origin of the coordinate system is at the cell center, the average value of each component of strains or strains gradient in Eq. (2) is equal to its value at the cell origin. Then, if $\langle\cdot\rangle$ stands for the averaging operator defined over the continuum cell, we have:

$\langle\alpha\rangle=\frac{1}{\left|S_{\mathrm{c}}\right|} \int_{S_{\mathrm{c}}} \alpha \mathrm{d} S_{\mathrm{c}}$

where $\left|S_{\mathrm{c}}\right|$ is the surface of the continuum cell, i.e. $\left|S_{\mathrm{c}}\right|=$ lh in our case (a unit thickness is assumed). Eq. (2) is now written as follows:

$$
\begin{aligned}
& u(x, y)=u_{0}+x\left\langle\epsilon_{x}\right\rangle+y\left(\frac{1}{2}\left\langle\gamma_{x y}\right\rangle-r_{0}\right)+x y\left\langle\epsilon_{x, y}\right\rangle \\
& v(x, y)=v_{0}+x\left(\frac{1}{2}\left\langle\gamma_{x y}\right\rangle+r_{0}\right)+y\left\langle\epsilon_{y}\right\rangle+x y\left\langle\epsilon_{y, x}\right\rangle
\end{aligned}
$$

\subsubsection{Double-bay lattice}

The average value over the cell is calculated from the intermediate results obtained for each bay. Let $\langle\cdot\rangle_{i}$ denote the averaging operator over the $i$ th bay; then, for two bays of the same length $l / 2$ we have:

$\langle\alpha\rangle=\left(\langle\alpha\rangle_{1}+\langle\alpha\rangle_{2}\right) / 2$

Defining $\bar{\alpha}$ as:

$\bar{\alpha}=\langle\alpha\rangle-\langle\alpha\rangle_{1}=\langle\alpha\rangle_{2}-\langle\alpha\rangle$

Eq. (3) becomes: 
$\bar{u}_{0}=\frac{l}{4}\left\langle\epsilon_{x}\right\rangle$

$\bar{v}_{0}=\frac{l}{4}\left(\frac{1}{2}\left\langle\gamma_{x y}\right\rangle+\left\langle r_{0}\right\rangle\right)$

$\frac{1}{2} \bar{\gamma}_{x y}-\bar{r}_{0}=\frac{l}{4}\left\langle\epsilon_{x, y}\right\rangle$

$\bar{\epsilon}_{y}=\frac{l}{4}\left\langle\epsilon_{y, x}\right\rangle$

In each bay, Eq. (7) can be used to express the displacement field as a function of $\langle\alpha\rangle$ and $\bar{\alpha}$, where $\alpha$ is one of the following items: $\left\{u_{0}, v_{0}, r_{0}, \epsilon_{x}, \epsilon_{y}, \gamma_{x y}, \epsilon_{x, y}, \epsilon_{y, x}\right\}$. The number of parameters can be reduced from 16 to 12 by eliminating $\bar{u}_{0}, \bar{v}_{0}, \frac{1}{2} \bar{\gamma}_{x y}-\bar{r}_{0}$ and $\bar{\epsilon}_{y}$ using Eq. (8).

\subsubsection{Comparison with other methods}

For the methods based on a Taylor series expansion, the strain and strain gradient parameters for the continuum are defined by their value at the cell center. In the case of the single-bay lattice, our approach is equivalent to the preceding ones, both for the displacement field (see Subsection 2.1.3) and for the continuum strain parameters.

For the multiple-bay lattice, the comparison is more difficult because of the difference between the two displacement fields. However, it can be noticed that the macroscopic strains used in our approach are not equal to their values at the cell center. Since we are interested in the overall behaviour of the continuum, the choice of averaging the macroscopic strain gradients seems to be better, as in the homogenization theory [10].

\subsection{Macroscopic stresses}

The effective stiffness of the lattice relates the macroscopic strains (defined in the previous section) to the macroscopic stresses. Two means can be used to calculate these macroscopic stresses.

\subsubsection{Beam analogy}

Let us consider a typical cell of the (single- or multiple-bay) lattice, as an element of a repetitive lattice subjected to external loads. The cell is in equilibrium with concentrated loads applied to the nodes at $x= \pm l / 2$. The loads are assumed to be zero at the other nodes of the boundary $y= \pm l / 2$, as shown in Fig. 3.

For such a beam-like lattice, the continuum model is a one-dimensional beam. Then, if the cell is considered as a beam of length $l$, the extensional force $N$ and the

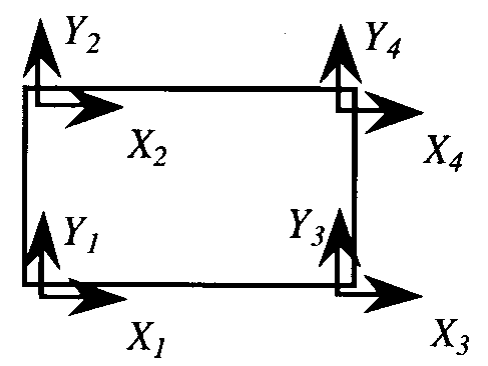

Fig. 3. The cell isolated from the repetitive lattice.

transverse shear force $T$ are constant along the beam, and the bending moment $M$ is linear. Therefore, one directly obtains the average value of the integrated stresses over the beam length:

$$
\langle N\rangle=X_{3}+X_{4}=-\left(X_{1}+X_{2}\right)=\frac{1}{l} \sum_{i} x_{i} X_{i}
$$

$$
\begin{aligned}
\langle T\rangle & =\frac{1}{l} \sum_{i} x_{i} Y_{i} \\
\langle M\rangle & =\frac{1}{2}\left(\frac{h}{2}\left(X_{2}-X_{1}\right)+\frac{h}{2}\left(X_{3}-X_{4}\right)\right) \\
& =-\frac{1}{l} \sum_{i} x_{i} y_{i} X_{i}
\end{aligned}
$$

where the summation is made from 1 to 4 .

It should be noticed that, after assuming the continuum to behave like a beam, the macroscopic integrated stresses are averaged over the beam length.

\subsubsection{Energy equivalence}

Another means of obtaining the macroscopic stresses is to equate the strain energy of the repeated discrete lattice and that of the continuum element. The energy equivalence is classically used in the substitute continuum approach, and also in the homogenization methods. It will enable us to characterize the macroscopic stresses as the dual quantities of the macroscopic strains.

\subsubsection{Single-bay lattice}

The finite element analysis of the lattice cell shown in Fig. 3 gives a system of the form:

$\left[K_{\mathrm{d}}\right]\left\{u_{\mathrm{d}}\right\}=\left\{F_{\mathrm{d}}\right\}$

where $\left[K_{\mathrm{d}}\right]$ is the stiffness matrix, $\left\{u_{\mathrm{d}}\right\}$ the vector of nodal displacements and $\left\{\mathrm{F}_{\mathrm{d}}\right\}$ the vector of equivalent nodal forces $\left(X_{i}, Y_{i}\right)$ (the subscript 'd' refers to the discrete lattice). Eq. (5) can be written at each node $i=1,4$ as follows: 
$\left\{\begin{array}{c}u_{i} \\ v_{i}\end{array}\right\}=\left[\Gamma_{i}\right]\left\{u_{\mathrm{c}}\right\}$

where $\left\{u_{\mathrm{c}}\right\}^{\mathrm{T}}=\left\{u_{0}, v_{0}, r_{0},\{E\}^{\mathrm{T}},\left\{E_{1}\right\}^{\mathrm{T}},\left\{E_{2}\right\}^{\mathrm{T}}\right\}$ (the subscript 'c' refers to the continuum) with

$\{E\}^{\mathrm{T}}=\left\{\left\langle\epsilon_{x}\right\rangle,\left\langle\gamma_{x y}\right\rangle,\left\langle-\epsilon_{x, y}\right\rangle\right\}$

$E_{1}=\left\langle\epsilon_{y}\right\rangle ; \quad E_{2}=\left\langle\epsilon_{y, x}\right\rangle$

and where the matrix $\left[\Gamma_{i}\right]$ is defined as:

$\left[\Gamma_{i}\right]=$

$$
\left[\begin{array}{cccccccc}
1 & 0 & -y_{i} & x_{i} & y_{i} / 2 & -x_{i} y_{i} & 0 & 0 \\
0 & 1 & x_{i} & 0 & x_{i} / 2 & 0 & y_{i} & x_{i} y_{i}
\end{array}\right]
$$

This matrix differs from the matrix $\left[T_{i}\right]$ given in Ref. [4] where quadratic terms in $x_{i}$ and $y_{i}$ are added because $\epsilon_{x, y}$ and $\epsilon_{y, x}$ appear in the coefficients of the polynomials representing the displacement field respectively in $v_{, x x}$ and $u_{, y y}$. Since the origin of the coordinate system is at the cell center, these terms are constant, and it can be derived that transformation of Eqs. (11) and (13) is equivalent to that proposed in Ref. [4].

Then, with $[\Gamma]^{\mathrm{T}}=\left[\left[\Gamma_{1}\right]^{\mathrm{T}},\left[\Gamma_{2}\right]^{\mathrm{T}},\left[\Gamma_{3}\right]^{\mathrm{T}},\left[\Gamma_{4}\right]^{\mathrm{T}}\right]$, we can write:

$\left\{u_{\mathrm{d}}\right\}=[\Gamma]\left\{u_{\mathrm{c}}\right\}$

and substituting Eq. (14) in Eq. (10) yields:

$[\Gamma]^{T}\left[K_{\mathrm{d}}\right][\Gamma]\left\{u_{\mathrm{c}}\right\}=[\Gamma]^{T}\left\{F_{\mathrm{d}}\right\}$

Denoting $\left\{F_{\mathrm{c}}\right\}=[\Gamma]^{\mathrm{T}}\left\{F_{\mathrm{d}}\right\}$, this vector is found to be:

$$
\begin{gathered}
\left\{F_{\mathrm{c}}\right\}^{T}=\left\{\sum_{i} X_{i}, \sum_{i} Y_{i}, \sum_{i}\left(-y_{i} X_{i}+x_{i} Y_{i}\right),\right. \\
\sum_{i} x_{i} X_{i}, \sum_{i}\left(y_{i} X_{i}+x_{i} Y_{i}\right) / 2-\sum_{i} x_{i} y_{i} X_{i}, \\
\left.\sum_{i} y_{i} Y_{i}, \sum_{i} x_{i} y_{i} Y_{i}\right\}
\end{gathered}
$$

Next, the strain energy on the discrete cell expressed in terms of the continuum parameters is given by:

$U_{\mathrm{d}}=\frac{1}{2}\left\{u_{\mathrm{c}}\right\}^{\mathrm{T}}\left\{F_{\mathrm{c}}\right\}$

On the other hand, the strain energy on the continuum (a unit thickness is assumed) is expressed as:
$U_{\mathrm{c}}=\frac{1}{2} \int_{S_{\mathrm{c}}} \overline{\bar{\sigma}}: \overline{\bar{\epsilon}} \mathrm{d} S_{\mathrm{c}}$

where $S_{\mathrm{c}}$ is the continuum cell surface.

Using Eq. (5), $U_{\mathrm{c}}$ can be re-written as:

$U_{\mathrm{c}}=\frac{1}{2}\left\{u_{\mathrm{c}}\right\}^{\mathrm{T}}\left\{F_{\sigma}\right\}$

where the vector $\left\{F_{\sigma}\right\}$ is found to be:

$$
\begin{aligned}
& \left\{F_{\sigma}\right\}^{\mathrm{T}}=\int_{S_{\mathrm{c}}}\left\{0,0,0, \sigma_{x x}, \sigma_{x y},\right. \\
& \left.\quad-\left(y \sigma_{x x}+x \sigma_{x y}\right), \sigma_{y y},\left(x \sigma_{y y}+y \sigma_{x y}\right)\right\} \mathrm{d} S_{\mathrm{c}}
\end{aligned}
$$

In order to calculate the components of $\left\{F_{\sigma}\right\}$, given that the continuum is a beam of axis $x$, we write:

$$
\begin{gathered}
\int_{S_{\mathrm{c}}} \sigma_{x x} \mathrm{~d} S_{\mathrm{c}}=\int_{-l / 2}^{l / 2}\left(\int_{-h / 2}^{h / 2} \sigma_{x x} \mathrm{~d} y\right) \mathrm{d} x \\
=\int_{-l / 2}^{l / 2} N(x) \mathrm{d} x=l\langle N\rangle
\end{gathered}
$$

In the same way, it can easily be seen that the fifth component of $\left\{F_{\sigma}\right\}$ is equal to $l\langle T\rangle$. In order to show that the sixth component of $\left\{F_{\sigma}\right\}$ is equal to $l\langle M\rangle$, let us prove that:

$\int_{S_{\mathrm{c}}} x \sigma_{x y} \mathrm{~d} S_{\mathrm{c}}=0$

Since the center of the cell is at $x=0$, it is sufficient to show that $\int_{-h / 2}^{h / 2} \sigma_{x y} \mathrm{~d} y$ is independent of $x$. To this end, the continuum is considered as a bidimensional domain in plane stress state, subjected to external loads on its boundary $x= \pm l / 2$ and with free edges at $y= \pm l / 2$. Then, using the equilibrium equation, we have:

$\frac{\partial}{\partial x}\left(\int_{-h / 2}^{h / 2} \sigma_{x y} \mathrm{~d} y\right)=-\int_{-h / 2}^{h / 2} \sigma_{y y, y} \mathrm{~d} y$

and this last integral is zero because of the boundary conditions.

From the energy equivalence $U_{\mathrm{d}}=U_{\mathrm{c}}$, and using Eqs. (17) and (19), one can derive:

$\left\{F_{\mathrm{c}}\right\}=\left\{F_{\sigma}\right\}$

As a result, it can be checked that the first three components of $\left\{F_{\mathrm{c}}\right\}$ are zero, which corresponds to the cell equilibrium. The next three relationships show that the energy equivalence enables us to defined the average integrated stresses as the dual quantities of the average strains. The last two components of $\left\{F_{\sigma}\right\}$ will be identified later.

It follows that $\left\{F_{\sigma}\right\}$ can be put in the form: 
$\left\{F_{\sigma}\right\}^{\mathrm{T}}=l\left\{0,0,0,\{\Sigma\}^{\mathrm{T}},\left\{F_{1}\right\}^{\mathrm{T}},\left\{F_{2}\right\}^{\mathrm{T}}\right\}$

with

$\{\Sigma\}^{\mathrm{T}}=\{\langle N\rangle,\langle T\rangle,\langle M\rangle\}$

$l F_{1}=\int_{S_{\mathrm{c}}} \sigma_{y y} \mathrm{~d} S_{\mathrm{c}}=\sum_{i} y_{i} Y_{i}$

$l F_{2}=\int_{S_{\mathrm{c}}}\left(x \sigma_{y y}+y \sigma_{x y}\right) \mathrm{d} S_{\mathrm{c}}=\sum_{i} x_{i} y_{i} Y_{i}$

It can be seen that the results are in agreement with those of Subsection 2.3.2.1 where the energy equivalence was not used: taking into account that

$\sum_{i}\left(-y_{i} X_{i}+x_{i} Y_{i}\right)=0$

we have:

$\sum_{i}\left(y_{i} X_{i}+x_{i} Y_{i}\right) / 2=\sum_{i} x_{i} Y_{i}$

\subsubsection{Double-bay lattice}

The modifications to the previous development for the case of a double-bay lattice are presented now. From the results of Section 2.2.2, the vector $\left\{u_{\mathrm{c}}\right\}$ can be noted:

$\left\{u_{\mathrm{c}}\right\}^{\mathrm{T}}=\left\{\left\{u^{*}\right\}^{\mathrm{T}},\{E\}^{\mathrm{T}},\left\{E^{*}\right\}^{\mathrm{T}},\left\{E_{1}\right\}^{\mathrm{T}},\left\{E_{2}\right\}^{\mathrm{T}}\right\}$

with

$\left\{u^{*}\right\}^{\mathrm{T}}=\left\{\left\langle u_{0}\right\rangle,\left\langle v_{0}\right\rangle,\left\langle r_{0}\right\rangle\right\} ; \quad\left\{E^{*}\right\}^{\mathrm{t}}=\left\{\bar{\epsilon}_{x}, \frac{1}{2} \bar{\gamma}_{x y}+\bar{r}_{0}\right\}$

$\{E\}^{\mathrm{T}}=\left\{\left\langle\epsilon_{x}\right\rangle,\left\langle\gamma_{x y}\right\rangle,\left\langle-\epsilon_{x, y}\right\rangle\right\}$

$\left\{E_{1}\right\}^{\mathrm{T}}=\left\{\left\langle\epsilon_{y}\right\rangle, \bar{\epsilon}_{x, y}, \bar{\epsilon}_{y, x}\right\} ; \quad\left\{E_{2}\right\}^{\mathrm{T}}=\left\{\left\langle\epsilon_{y, x}\right\rangle\right\}$

Then, expressing Eq. (5) in the global coordinate system (the origin of which is at the cell center), the matrix $[\Gamma]$ defined in Eq. (14) can be found as follows:

- for the first-bay nodes (the superscript indicates the bay number):

$\left\{\begin{array}{c}u_{i}^{1} \\ v_{i}^{1}\end{array}\right\}=\left[\Gamma_{i}^{1}\right]\left\{u_{\mathrm{c}}\right\}$

with

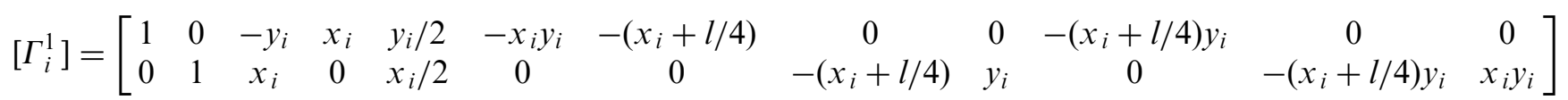

- for the second-bay nodes, similar expressions hold, with the four terms including $-\left(x_{i}+l / 4\right)$ changed into $+\left(x_{i}-l / 4\right)$.

We obtain $[\Gamma]^{\mathrm{T}}=\left[\left[\Gamma_{1}^{1}\right]^{\mathrm{T}},\left[\Gamma_{2}^{1}\right]^{\mathrm{T}},\left[\Gamma_{3}^{2}\right]^{\mathrm{T}},\left[\Gamma_{4}^{2}\right]^{\mathrm{T}},\left[\Gamma_{5}^{1}\right]^{\mathrm{T}}\right.$, $\left.\left[\Gamma_{6}^{1}\right]^{\mathrm{T}}\right]$, where the nodes $1-4$ are shown in Fig. 3 and the nodes 5 and 6 are located at the junction of the two bays. Since there is no external load on these two interior nodes, the calculation of $\left\{F_{\mathrm{c}}\right\}=[\Gamma]^{\mathrm{T}}\left\{F_{\mathrm{d}}\right\}$ yields:

$$
\begin{aligned}
\left\{F_{\mathrm{c}}\right\}^{\mathrm{T}}= & \left\{\sum_{i} X_{i}, \sum_{i} Y_{i} \sum_{i}\left(-y_{i} X_{i}+x_{i} Y_{i}\right),\right. \\
& \sum_{i} x_{i} X_{i}, \frac{1}{2} \sum_{i}\left(y_{i} X_{i}+x_{i} Y_{i}\right), \\
& -\sum_{i} x_{i} y_{i} X_{i}, \frac{l}{4} \sum_{i} X_{i}, \frac{l}{4} \sum_{i} Y_{i}, \sum_{i} y_{i} Y_{i}, \frac{l}{4} \sum_{i} y_{i} X_{i}, \\
& \left.\frac{l}{4} \sum_{i} y_{i} Y_{i}, \sum_{i} x_{i} y_{i} Y_{i}\right\}
\end{aligned}
$$

where the summation is made from 1 to 4 .

The energy equivalence gives the eight relations in Eqs. (16), (20), (24), (26) and four new relationships which are not reported here. The vector $\left\{F_{\sigma}\right\}$ can then be defined as:

$\left\{F_{\sigma}\right\}^{\mathrm{T}}=l\left\{0,0,0,\{\Sigma\}^{\mathrm{T}},\left\{F^{*}\right\}^{\mathrm{T}},\left\{F_{1}\right\}^{\mathrm{T}},\left\{F_{2}\right\}^{\mathrm{T}}\right\}$

with

$\{\Sigma\}^{\mathrm{T}}=\{\langle N\rangle,\langle T\rangle,\langle M\rangle\} ; \quad\left\{F^{*}\right\}^{\mathrm{T}}=\{0,0\}$

$l\left\{F_{1}\right\}^{\mathrm{T}}=\sum_{i}\left\{y_{i} Y_{i}, \frac{l}{4} y_{i} X_{i}, \frac{l}{4} y_{i} Y_{i}\right\}$

$l F_{2}=\sum_{i} x_{i} y_{i} Y_{i}$

\subsubsection{Comments on the continuum stresses}

In Section 2.3, it is shown that the energy equivalence makes it possible to calculate the macroscopic integrated stresses. It must be noticed that in the literature, the developments are usually focused on the strain and kinetic energies [1-6] and no special attention is paid to the integrated stresses. It will be seen in Section 4 that the knowledge of these efforts allows a better understanding of the assumptions used for the computation of the equivalent continuum parameters. 


\section{Matrix formulation}

After suppressing the rows and columns corresponding to the rigid body terms, Eqs. (15), (24) and (25) lead to:

$$
\left[\begin{array}{lll}
K_{11}^{1} & K_{12}^{1} & K_{13}^{1} \\
K_{21}^{1} & K_{22}^{1} & K_{23}^{1} \\
K_{31}^{1} & K_{32}^{1} & K_{33}^{1}
\end{array}\right]\left\{\begin{array}{l}
\{E\} \\
E_{1} \\
E_{2}
\end{array}\right\}=l\left\{\begin{array}{l}
\{\Sigma\} \\
F_{1} \\
F_{2}
\end{array}\right\}
$$

for a single-bay lattice, and in the same way, the relation:

$$
\left[\begin{array}{llll}
K_{11}^{2} & K_{12}^{2} & K_{13}^{2} & K_{14}^{2} \\
K_{21}^{2} & K_{22}^{2} & K_{23}^{2} & K_{24}^{2} \\
K_{31}^{2} & K_{32}^{2} & K_{33}^{2} & K_{34}^{2} \\
K_{41}^{2} & K_{42}^{2} & K_{43}^{2} & K_{44}^{2}
\end{array}\right]\left\{\begin{array}{l}
\{E\} \\
\left\{E^{*}\right\} \\
\left\{E_{1}\right\} \\
\left\{E_{2}\right\}
\end{array}\right\}=l\left\{\begin{array}{l}
\{\Sigma\} \\
\{0\} \\
\left\{F_{1}\right\} \\
\left\{F_{2}\right\}
\end{array}\right\}
$$

is obtained for a double-bay lattice.

\section{Computation of beam parameters}

The problem is to find $\left[D^{\text {hom }}\right]$ such that:

$$
\{\Sigma\}=\left[D^{\text {hom }}\right]\{E\}
$$

Three different methods are proposed herein, consisting of eliminating the strain and stress terms in Eqs. (35) and (36) that are not involved in the Timoshenkobeam theory.

\subsection{The periodic solution}

Due to the periodicity of the repetitive lattice, the strains and internal efforts are locally periodic in the lattice. This also holds for the strains and stresses in the equivalent continuum which is periodic too. Then:

$$
\left\{X_{1}, X_{2}, Y_{1}, Y_{2}\right\}=-\left\{X_{3}, X_{4}, Y_{3}, Y_{4}\right\}
$$

and $\epsilon_{y}$ will take the same value on the opposite sides of the cell. Thus, it turns out that $\left\{\mathrm{E}_{2}\right\}=\left\{\mathrm{F}_{1}\right\}=0$. As a result:

$D_{\mathrm{Per}}^{\text {hom }}=\frac{1}{l}\left(K_{11}-K_{12} K_{22}^{-1} K_{21}\right)$

where $K_{i j}=K_{i j}^{1}, i, j=1,2$ for the single-bay lattice, and

$$
\begin{gathered}
K_{11}=K_{11}^{2} ; \quad\left[K_{12}\right]=\left[K_{12}^{2} K_{13}^{2}\right] ; \quad\left[K_{21}\right]=\left[\begin{array}{c}
K_{21}^{2} \\
K_{31}^{2}
\end{array}\right] ; \\
{\left[K_{22}\right]=\left[\begin{array}{ll}
K_{22}^{2} & K_{23}^{2} \\
K_{32}^{2} & K_{33}^{2}
\end{array}\right]}
\end{gathered}
$$

for the double-bay lattice.

This method of computation of the beam parameters is equivalent to that proposed in Ref. [2]. In the above mentioned paper, the strain components corresponding to $\left\{E_{2}\right\}$ are set to zero so as to ensure the compatibility between the repeated elements, which is of course equivalent to the strain periodicity. On the other hand, $\left\{F_{1}\right\}$ is set to zero according to the Timoshenko-beam theory, because this force is associated with the strain component $\epsilon_{y}$.

Therefore, we have presented a rigorous way of obtaining the effective coefficients of the beam, and the lattice periodicity is taken into account. However, it is interesting to show that approximate methods can be derived from the matrix formulation of Section 3, and compare them with other methods discussed in the literature.

\subsection{A force method}

Another way of obtaining the overall beam behaviour is to assume that $\left\{F_{1}\right\}=\left\{F_{2}\right\}=\{0\}$, which gives:

$D_{F}^{\text {hom }}=\frac{1}{l}\left(K_{11}^{*}-K_{12}^{*}\left(K_{22}^{*}\right)^{-1} K_{21}^{*}\right)$

where $K_{i j}^{*}, i, j=1,2$ is given by a formula similar to Eq. (39) for the single-bay lattice, and for the double bay lattice:

$$
\begin{aligned}
& K_{11}^{*}=K_{11}^{2} ; {\left[K_{12}^{*}\right]=\left[K_{12}^{2} K_{13}^{2} K_{14}^{2}\right] } \\
& {\left[K_{21}^{*}\right]=} {\left[\begin{array}{l}
K_{21}^{2} \\
K_{31}^{2} \\
K_{41}^{2}
\end{array}\right] ; } \\
& {\left[K_{22}^{*}\right]=\left[\begin{array}{lll}
K_{22}^{2} & K_{23}^{2} & K_{24}^{2} \\
K_{32}^{2} & K_{33}^{2} & K_{34}^{2} \\
K_{42}^{2} & K_{43}^{2} & K_{44}^{2}
\end{array}\right] }
\end{aligned}
$$

Such a method amounts to eliminating, through static condensation, all the strain parameters which do not appear in the macroscopic strains $\{E\}$ of the continuum. This approach has already been applied in Refs. [3-4].

Another means of determining the matrix $D$ hom is to submit the lattice (with the help of the matrix $[\Gamma]$ 

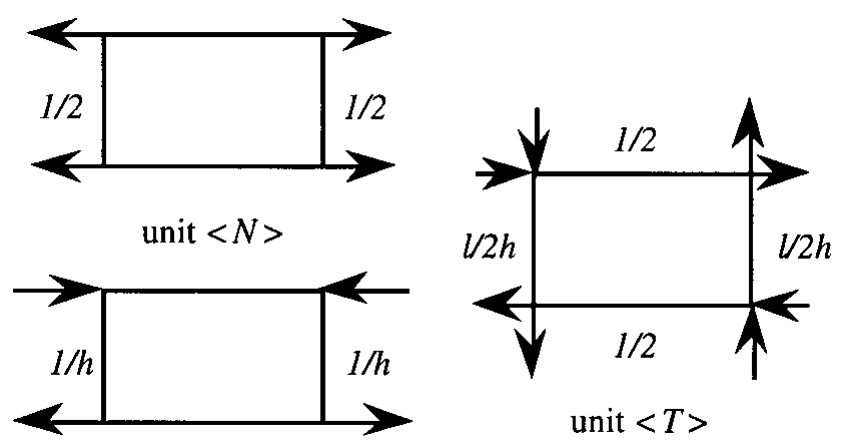

unit $\langle T>$
Fig. 4. The force method loading cases (single-bay lattice).

defined in Subsection 2.3.2.1 and 2.3.2.2) to three different loading cases corresponding to a unit load, respectively, in $\langle N\rangle,\langle T\rangle$ or $\langle M\rangle$ with $\left\{F_{1}\right\}=\left\{F_{2}\right\}=\{0\}$. Then, from the inverse of the matrix $[\Gamma]$ and the vector of the nodal displacements, $\{E\}$ is calculated and yields the inverse of $D_{\mathrm{F}}^{\text {hom }}$, column by column. For the single bay lattice, the three loading cases are shown in Fig. 4.

These loading cases can be found in Ref. [6]. One of the merits of the formulation used herein is to show that, because of the regularity of $[\Gamma]$, there is only one loading case for a given $\{\Sigma\}$ under the assumption that $\left\{F_{1}\right\}=\left\{F_{2}\right\}=\{0\}$. For a double-bay lattice and for the loading case correspond to a unit $\langle T\rangle$, there are external loads exerted on the nodes at the junction of the two bays.

Lastly, it must also be mentioned that such a method based on the loading cases is cumbersome compared to Eq. (40). However, it is very easy to use with a standard finite element program and it gives a physical interpretation to the hypothesis $\left\{F_{1}\right\}=\left\{F_{2}\right\}=\{0\}$, which amounts to assuming the load distribution on the lattice boundary for a given $\{\Sigma\}$.

\subsection{A displacement method}

The dual standpoint of the previous force method is to assume that $\left\{E_{1}\right\}=\left\{E_{2}\right\}=\{0\}$, which yields:

$D_{\mathrm{D}}^{\text {hom }}=\frac{1}{l} K_{11}^{1}$

for the single-bay lattice and

$D_{\mathrm{D}}^{\text {hom }}=\frac{1}{l}\left(K_{11}^{2}-K_{12}^{2}\left(K_{22}^{2}\right)^{-1} K_{21}^{2}\right)$

for the double-bay lattice.

Again, the matrix $D_{\mathrm{D}}^{\text {hom }}$ can be computed after sol- ving a local problem on the lattice cell with displacement boundary conditions corresponding to a unit $\left\langle\epsilon_{x}\right\rangle,\left\langle\gamma_{x y}\right\rangle$ or $\left\langle-\epsilon_{x, y}\right\rangle$ with $\left\{E_{1}\right\}=\left\{E_{2}\right\}=\{0\}$. The vector $\{\Sigma\}$ is then calculated from the reactions $\left(X_{i}, Y_{i}\right)$ at each node (using Eqs. (16) and (24)).

For a single-bay lattice, the boundary conditions for each loading case are easily derived from the matrix $[\Gamma]$; they are shown in Fig. 5. For a single-bay lattice, with $\left\{E_{1}\right\}=\left\{E_{2}\right\}=\{0\}$, we take six parameters to represent the displacement field. Moreover, the left and right sections of the beam remain plane, with $\epsilon_{y}=0$. Then, the kinematical assumptions are equivalent to the approach presented in Ref. [5]. However, the method proposed in the paper mentioned above is restricted to a one-bay lattice, and the equivalent beam parameters are not obtained directly, but through the comparison of finite element stiffness matrices of the lattice and the extended Timoshenko beam models.

For the double-bay lattice, if $\{E *\}=0$ was assumed, the displacement boundary conditions shown in Fig. 5 would be determined, but this assumption is useless, because $\{F *\}=0$ holds. We have therefore:

$\left\{E^{*}\right\}=-\left(K_{22}^{2}\right)^{-1} K_{21}^{2}\{E\}$

from which boundary conditions for a given $\{E\}$ can be obtained. It can be checked that a zig-zag pattern can occur for each loading case. The last remarks in Section 4.2 are also valid for the displacement method.

\subsection{Comments on the previous methods}

First, one should bear in mind that in the case of the multiple-bay lattice, the initial system of equations given in Eqs. (34) or (35) is different from that proposed in Refs. [1-4]. With our approach, not only are the displacements written differently, but also the strains and integrated stresses which are averaged over the cell (these differences vanish for a single-bay lattice, see Section 2.2.3).

As mentioned previously, the periodic approach for the computation of the effective beam properties is equivalent to the method initially proposed in Ref. [2] by Noor and his coworkers. The static condensation was used later by Noor et al. [3], and Dow [4], and is equivalent to the procedure of Sun et al. [6], and to the force methods presented in this paper. Lastly, there are similarities between the approach of Lee [5] and the displacement method given herein, but the latter is more general.

Moreover, it is worth noticing that the characterization of the right-hand member of the system of equations (i.e. the macroscopic integrated stresses) enables us: 


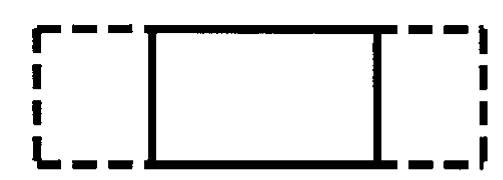

unit $\left\langle\varepsilon_{x}>\right.$

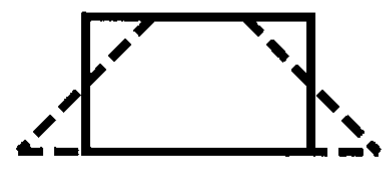

unit $\left\langle-\varepsilon_{x, y}>\right.$

Fig. 5. Boundary conditions of the displacement method (single-bay lattice).

- to give an estimate of the internal truss forces in terms of the resultant forces acting on the equivalent beam section (from Eqs. (34) or (35) and the matrix $[\Gamma])$;

- to obtain a unifying approach for deriving different methods for computing the effective beam properties;

- to gain insight into the assumptions of each method.

Thus, it can be concluded that the periodic approach is the most accurate way of obtaining the effective stiffness of the continuum, because it is the only method which really takes the periodicity of the repetitive lattice into account (in this way, it can be said that $\left\{E_{2}\right\}=\left\{F_{1}\right\}=0$ are not assumptions). Consequently, the force and displacement methods are approximate, and their drawback is to lead to equivalent beam properties which depend on the cell choice.

Besides, the approaches used in the present work can be compared with homogenization methods for periodic materials. Indeed, the force method, the periodic approach and the displacement method are equivalent to the localization problems found in the homogenization theory, with respectively uniform stress, periodic, or uniform strain boundary conditions [10]. Furthermore, as can be seen in Section 5 hereafter, the strain energies corresponding to each method are classified in the same order as in homogenization.

\section{Comparison between the three methods}

In Section 4, three different methods for the computation of beam parameters have been proposed. The aim of this section is to examine the possibility of comparing the results given by each method.
Let us first prove the following lemma:

Lemma 5.1. let $K_{i j}, i, j=1,2$ be submatrices of a positive definite symmetric stiffness matrix $K$. Then the following inequality holds:

$\forall\{E\},\{E\}^{\mathrm{T}}\left(K_{11}-K_{12} K_{22}^{-1} K_{21}\right)\{E\} \leq\{E\}^{\mathrm{T}} K_{11}\{E\}$

Proof. the result is a direct consequence of the symmetry and the definite positiveness of the matrix $K$. One can write:

$\{E\}^{\mathrm{T}} K_{12} K_{22}^{-1} K_{21}\{E\}=\left(K_{21}\{E\}\right)^{\mathrm{T}} K_{22}^{-1}\left(K_{21}\{E\}\right)$

which proves the lemma, because the second term of the equality is clearly positive.

Using the lemma, one can show that:

$\forall\{E\},\{E\}^{\mathrm{T}} D_{\mathrm{Per}}^{\text {hom }}\{E\} \leq\{E\}^{\mathrm{T}} D_{\mathrm{D}}^{\text {hom }}\{E\}$

$\forall\{E\},\{E\}^{\mathrm{T}} D_{\mathrm{F}}^{\text {hom }}\{E\} \leq\{E\}^{\mathrm{T}} D_{\mathrm{Per}}^{\text {hom }}\{E\}$

For the second inequality, the lemma is used after the static condensation of $\left\{E_{1}\right\}$ (and $\{E *\}$ for the doublebay lattice). A system of equations is then obtained with $\{E\}$ and $\left\{E_{2}\right\}$ and the submatrix of indices 11 is found to be $l D$ Per .

As shown in Section 4.4, the periodic approach is the most accurate method for calculating the equivalent continuum properties; it follows from Eq. (47) that the force method underestimates the stiffnesses, while the displacement method overestimates it.

\section{Numerical examples}

The methods presented previously are now illus-

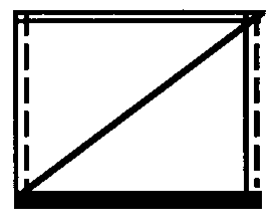

Fig. 6. The single-laced single-bay lattice. 
Table 1

Material properties and geometric dimensions of the single-laced single-bay lattice

\begin{tabular}{lllll}
\hline Lattice members & $E\left(\mathrm{~N} / \mathrm{m}^{2}\right)$ & $L(\mathrm{~m})$ & $S\left(\mathrm{~m}^{2}\right)$ & Designation \\
\hline Long bar & $71.7 \times 10^{9}$ & 7.5 & $8 \times 10^{-5}$ & $=$ \\
Diagonal bar & $71.7 \times 10^{9}$ & 9.0 & $4 \times 10^{-5}$ & - \\
Batten & $71.7 \times 10^{9}$ & 5.0 & $6 \times 10^{-5}$ & - \\
\hline
\end{tabular}

trated through examples of single- or double-bay lattices. The equivalent beam properties are denoted as follows:

$$
\left\{\begin{array}{l}
\langle N\rangle \\
\langle T\rangle \\
\langle M\rangle
\end{array}\right\}=\left[\begin{array}{lll}
E S & C_{1} & C_{2} \\
C_{1} & G S & C_{3} \\
C_{2} & C_{3} & E I
\end{array}\right]\left\{\begin{array}{l}
\left\langle\epsilon_{x}\right\rangle \\
\left\langle\gamma_{x y}\right\rangle \\
\left\langle-\epsilon_{x, y}\right\rangle
\end{array}\right\}
$$

\subsection{Single-bay lattice}

Let us first consider a single-laced single-bay lattice shown in Fig. 6; its dimensions and material properties are given in Table 1 . This lattice is analyzed with the three methods presented in Section 4, using a cell with two vertical trusses of area $S / 2$. The results are shown in Table 2.

It can be checked that the force method results coincide with those given in Ref. [6] (the sign of $C_{2}$ is different because of the rotation convention). We can notice that the force method and the periodic approach give the same results for this example. Examining the stiffness matrix of Eq. (34) shows that $K_{13}^{1}=K_{31}^{1}=0$ and that the submatrix $K_{33}^{1}$ is diagonal, hence this equality. Besides, the results are in agreement with the inequalities of Eq. (47).

If a cell with only one vertical truss of area $S$ is used, the periodic and displacement methods provide the same results as those of Table 2. On the other hand, the force method gives very different results from those obtained with the cell with two vertical trusses of area $S / 2$, especially for the second row of the stiffness matrix, which is found to be zero, due to the fact that the loading case corresponding to the unit shear force leads to a vertical load applied at a node which is only connected with a horizontal bar.

The latter result emphasizes the importance of the approximations made in the force method to derive the equivalent beam properties. However, from the results of Table 2, it is not possible to judge which is better between the periodic and the displacement approaches. Therefore, a direct analysis of the actual lattice structure with 11 bays is made, with loadings corresponding to a unit extensional force, a transverse shear force and a bending moment, through the boundary conditions shown in Fig. 4. Then for each loading case, the macroscopic strains and then a compliance matrix are derived from the nodal displacements of the central bay of the lattice; and the stiffness matrix giving the effective beam properties is finally computed. The results show that the non-zero stiffness coefficients obtained with the periodic approach are within $0.02 \%$ of those of the discrete models, which confirms that this method is the most rigorous means of deriving the equivalent beam properties. Moreover,

Table 2

Equivalent beam properties

\begin{tabular}{lrrr}
\hline Methods & Force & Periodic & Displacement \\
\hline$E S\left(10^{7} \mathrm{~N}\right)$ & 2.013 & 2.013 & 2.029 \\
$G S\left(10^{5} \mathrm{~N}\right)$ & 6.592 & 6.592 & 7.343 \\
$E I\left(10^{7} \mathrm{Nm}^{2}\right)$ & 11.651 & 11.651 & 11.651 \\
$C_{1}\left(10^{5} \mathrm{~N}\right)$ & 9.889 & 9.889 & 11.014 \\
$C_{2}\left(10^{7} \mathrm{Nm}\right)$ & 1.793 & 1.793 & 1.793 \\
$C_{3}\left(10^{7} \mathrm{Nm}\right)$ & 0.000 & 0.000 & 0.000 \\
\hline
\end{tabular}




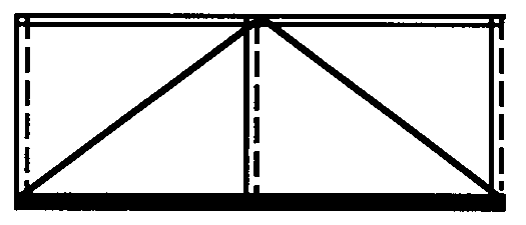

Fig. 7. A single-laced double-bay lattice.

for a cantilever repeated element loaded with an axial load, a shear load, and a pure moment, the continuum model based on the periodic solution is found to be the most accurate.

\subsection{Double-bay lattice}

\subsubsection{A test problem}

The first step consists of showing the reliability and the accuracy of the displacement field proposed herein by studying a double-bay lattice made of two identical one-bay lattices described in Section 6.1.

Then, with the expression of displacements given in Section 2.2.2, the equivalent beam parameters are calculated. It is possible to verify that for the three methods, the results exactly match those of Table 2 .

As a second step, the example is taken to illustrate a method in which a Taylor series expansion is used to write the displacement field [4] given by:

$$
\begin{aligned}
u(x, y)= & u_{0}+x \epsilon_{x}+y\left(\frac{1}{2} \gamma_{x y}-r_{0}\right)+x y \epsilon_{x, y}+\frac{x^{2}}{2} \epsilon_{x, x} \\
& +\frac{x^{2} y}{2} \epsilon_{x, x y}
\end{aligned}
$$

$$
\begin{aligned}
v(x, y)= & v_{0}+x\left(\frac{1}{2} \gamma_{x y}+r_{0}\right)+y \epsilon_{y}+x y \epsilon_{y, x} \\
& +\frac{x^{2}}{2}\left(\gamma_{x y, x}-\epsilon_{x, y}\right)+\frac{x^{2} y}{2} \epsilon_{y, x x}
\end{aligned}
$$

where all the rigid body displacements, strains and strain gradients are taken at the cell center.

As mentioned in Section 4.4, one can easily check that, for a single-bay lattice, the method based on the Taylor series expansion is equivalent to that proposed here. Then, this method also gives the results of Table 2.

For a double-bay lattice, from Eq. (49), we can define a transformation matrix between the discrete nodal displacements and the vector of the rigid body

Table 4

Equivalent beam properties

\begin{tabular}{lrcrr}
\hline Methods & Force & Periodic & Displacement & Identification \\
\hline$E S\left(10^{7} \mathrm{~N}\right)$ & 1.864 & 1.864 & 1.864 & 1.864 \\
$G S\left(10^{5} \mathrm{~N}\right)$ & 6.325 & 6.651 & 6.745 & 6.509 \\
$E I\left(10^{7} \mathrm{Nm}^{2}\right)$ & 11.651 & 11.651 & 11.651 & 11.651 \\
$C_{1}\left(10^{5} \mathrm{~N}\right)$ & 0.000 & 0.000 & 0.000 & 0.000 \\
$C_{2}\left(10^{7} \mathrm{Nm}\right)$ & 1.793 & 1.793 & 1.793 & 1.792 \\
$C_{3}\left(10^{7} \mathrm{Nm}\right)$ & 0.000 & 0.000 & 0.000 & 0.000 \\
\hline
\end{tabular}

Table 3

Equivalent beam properties

\begin{tabular}{lrrr}
\hline Methods & Force & Periodic & Displacement \\
\hline$E S\left(10^{7} \mathrm{~N}\right)$ & 2.000 & 2.000 & 2.029 \\
$G S\left(10^{5} \mathrm{~N}\right)$ & 6.029 & 6.029 & 7.343 \\
$E I\left(10^{7} \mathrm{Nm}^{2}\right)$ & 11.651 & 11.651 & 11.651 \\
$C_{1}\left(10^{5} \mathrm{~N}\right)$ & 9.044 & 9.044 & 11.014 \\
$C_{2}\left(10^{7} \mathrm{Nm}\right)$ & 1.793 & 1.793 & 1.793 \\
$C_{3}\left(10^{7} \mathrm{Nm}\right)$ & 0.000 & 0.000 & 0.000 \\
\hline
\end{tabular}


displacements, strains and strain gradients at the cell center. Then, the strain components are partitioned as in Eq. (27) with:

$$
\begin{aligned}
& \left\{u^{*}\right\}^{\mathrm{T}}=\left\{u_{0}, v_{0}, r_{0}\right\} ; \quad\left\{E^{*}\right\}^{\mathrm{T}}=\left\{\epsilon_{x, x}, \gamma_{x y, x}-\epsilon_{x, y}\right\} \\
& \{E\}^{\mathrm{T}}=\left\{\epsilon_{x}, \gamma_{x y},-\epsilon_{x, y}\right\} \\
& \left\{E_{1}\right\}^{\mathrm{T}}=\left\{\epsilon_{y}, \epsilon_{x, x y}, \epsilon_{y, x x}\right\} ; \quad\left\{E_{2}\right\}^{\mathrm{T}}=\left\{\epsilon_{y, x}\right\}
\end{aligned}
$$

It is therefore possible to apply the methods proposed in Section 4 to compute the equivalent beam parameters.

The results obtained for the particular double-bay lattice considered herein are shown in Table 3. As can be seen, except for the displacement method, these results deviate from the results of Table 2, with a difference which reaches $10 \%$ on $G S$ and $C_{1}$.

This discrepancy is not very large, but illustrates that the Taylor series expansion method gives results which depend on the way this single-bay lattice is treated (with a method developed for a one- or a doublebay lattice). The error results from the expression used in Eq. (49) for the displacements, where terms in $x^{2}$ and $x^{2} y$ appear.

The previous drawback (dependence of the results on the cell choice) does not appear with the displacement field proposed herein, based on piecewise linear functions.

\subsubsection{A single-laced double-bay lattice}

A single-laced double-bay lattice as shown in Fig. 7 is now taken as an example. Following the procedure presented in Section 2.2.2, the equivalent beam properties shown in Table 4 are obtained. It is noticed that the only difference between the three methods is the equivalent shear stiffness, which is classified as in Eq. (47).

We also present the results found with the method based on the displacement given in Eq. (49). The results are shown in Table 4, except for the equivalent shear stiffness which is: $5.459 \times 10^{5} \mathrm{~N}, 5.701 \times 10^{5} \mathrm{~N}$, and $6.745 \times 10^{5} \mathrm{~N}$, for the force, periodic and displacement methods, respectively.

In order to evaluate the accuracy of the stiffness coefficients obtained, the procedure described in Section 6.1 to identify the effective beam parameters was carried out. The results are given in the last column of Table 4, and show that the periodic method gives the most accurate solution.

\section{Conclusions}

A simple approach to the representation of repetitive beam-like lattice trusses by an equivalent continuum has been presented. The procedure is based on the energy equivalence between lattice and continuum models, and attempts are made to improve the accuracy of the displacements approximation in methods using polynomials. A piecewise linear displacement field is introduced.

Moreover, some features of the homogenization theory for periodic materials are used, e.g. the averaged strains and integrated stresses are taken as continuum parameters, and the lattice periodicity is used to determine the equivalent beam properties. The general formulation presented in this paper extends methods which have already been given in the literature to calculate the continuum properties. The assumptions made in these methods are pointed out, especially for the widely used method based on static condensation.

The numerical results given illustrate the reliability of the present method. The method has only been applied for the planar static analysis of beam-like lattice trusses, but it also can be used for three-dimensional beam- or plate-like lattice trusses in dynamic analysis. A similar method can also be developed for the study of rigid-joint lattices, with a displacement field written with third-order polynomials.

\section{References}

[1] Noor AK. Continuum modeling for repetitive lattice structures. Appl Mech Rev 1988;41(7):285-96.

[2] Noor AK, Anderson MS, Greene WH. Continuum models for beam- and platelike lattice structures. AIAA J 1978;16(12):1219-28.

[3] Noor AK, Russel WC. Anisotropic continuum models for beamlike lattice trusses. Comput Meth Appl Mech Engng 1986;57:257-77.

[4] Dow JO, Huyer SA. Continuum models of space station structures. J Aerospace Engng 1989;2(4):220-38.

[5] Lee U. Dynamic continuum modeling of beamlike space structures using finite element matrices. AIAA J 1990;28(4):725-31.

[6] Sun CT, Liebbe SW. Global-local approach to solving vibration of large truss structures. AIAA J 1990;28(2):303-8.

[7] Verna P. Modélisation des structures discrètes par homogénéisation: cas des treillis. Ph.D. thesis (in French), Institut National Polytechnique de Grenoble 1991.

[8] Renton JD. The beam-like behavior of space trusses. AIAA J 1984;22(2):273-80.

[9] Tollenaere H. Modéles bidimensionnels de tissés. Homogénéisation des treillis en vibrations libres. Ph.D. 
thesis (in French), Institut National Polytechnique de Grenoble 1994.

[10] Suquet P, Elements of homogenization for inelastic solid mechanics. In: Sanchez-Palencia E, Zaoui A, editors. Homogenization techniques for composite media. Lecture Notes in Physics, 272. Berlin: Springer, 1985. p. 194-278. 